\title{
Crack-tip force method for computing energy release rate in delaminated plates
}

\author{
Oung Park ${ }^{1}$, Bhavani V. Sankar* \\ Department of Aerospace Engineering, Mechanics and Engineering Science, 231 Aerospace Building, P.O. Box 116250, \\ University of Florida, Gainesville, FL 32611-6250, USA
}

\begin{abstract}
A new method called the crack-tip force method (CTFM) is derived for computing the energy release rate in delaminated beams and plates. In this method the delaminated plate is divided into two laminates on either side of the plane of delamination. The interaction forces, called crack-tip forces, between the sub-laminates at the crack-tip are computed. The energy release rate is expressed as a quadratic function of the crack-tip forces and the plate compliance coefficients. The CTFM is compared to the virtual crack closure technique (VCCT) as well as to a previously derived method called the strain energy density method using double cantilevered beam specimens as examples. The CTFM is found to be very efficient as the crack-tip forces are part of the solution of finite element analysis of delaminated plates, and they can be readily used to compute the point-wise energy release rate along the delamination front. (C) 2002 Elsevier Science Ltd. All rights reserved.
\end{abstract}

Keywords: Composite laminates; Crack-tip force method; Delamination; Energy release rate; Laminated plates; Virtual crack closure technique

\section{Introduction}

Delamination is a prevalent failure mechanism in laminated composite structures. Fracture mechanics concepts have been successfully applied to predict the loads at which the delamination will propagate and also for predicting their stability. Energy release rate $(G)$ has been found to be an useful measure in the analysis of delaminations even in dynamic situations such as impact $[1,2]$. The cost of computations dictate that beam/plate theories be used in computing $G$. Thus there is a need for efficient methods of computing $G$ and also methods for separation of fracture modes. Such beam theories have been routinely used in the analysis of fracture specimens, e.g., double cantilever beam, end notch flexure, etc. It should be noted that beam theories work well only when the delamination length is much longer than the thickness of the cracked sub-laminates. The same could be said of plate delaminations also. If the beam/plate theories can estimate the total strain energy in the delaminated structure with reasonable accuracy, then the same

\footnotetext{
${ }^{*}$ Corresponding author. Tel.: +1-352-392-6749; fax: +1-352-3927303.

E-mail address: sankar@ufl.edu (B.V. Sankar).

${ }^{1}$ Graduate Student, currently with Lexel Engineering, Flint, MI, USA.
}

theory can be used to estimate $G$ also. Such a condition exist when the delamination size is much larger than the plate thickness and plate theory solutions are valid at points away from the crack front. As the cracked ligaments become shorter the beam solutions deviate from the elastic fracture mechanics solutions and thus need corrections. Such corrections have been proposed by many researchers $[3,4]$.

In the present study three methods of computing $G$ in beam/plate-like structures are discussed. A new method called the crack-tip force method (CTFM) is derived and compared to the standard virtual crack closure technique (VCCT). In the context of a laminated plate, the point-wise $G$ along the delamination front is derived from the crack-tip forces.

\section{Energy release rate in laminated beams}

In this section we derive three methods of computing $G$ in a laminated beam. The beam example is used to minimize the complexity of derivations, but the methods can be extended to delaminated plates easily. We use shear deformable beam/plate theories throughout the study. In the case of beams the width is assumed to be unity. 


\subsection{Zero-volume J-Integral}

Consider a portion of the delaminated beam as shown in Fig. 1. We will assume that the delamination length or crack length $a \gg h$, the thickness of the thicker sub-laminate. We can use the $J$-Integral to compute the energy release rate $G$, if the path of the integral $A B C D E F$ shown in Fig. 1 is away from the crack-tip and the beam theory stresses along this path are reasonably accurate compared to the exact elasticity solutions. The $J$-Integral is defined as [5]

$J=\int_{\Gamma}\left(U_{0} n_{x}-\sigma_{i j} n_{j} u_{i, x}\right) \mathrm{d} s \quad i=1,2, \quad j=1,2$,

where $U_{0}$ is the strain energy density, $\sigma_{i j}$ and $u_{i}$ are, respectively, the stress and displacement components, and $n_{i}$ are the direction cosines of the outward normal along the path $\Gamma$. We will use the indices $i$ and $j$ or $x$ and $z$ interchangeably according to convenience. Further, summation is performed over repeated indices. We note that the $J$-Integral will vanish along the two horizontal paths $B C$ and $D E$, because along these two paths the direction cosine $n_{x}$ is equal to zero, and also the tractions given by $\sigma_{i j} n_{j}$ vanish. Hence the Integral is given as the sum of integrals along the three vertical paths: $A B, C D$ and $E F$. Next we will show that these vertical paths can be moved very close to the crack-tip without losing any accuracy in $G$.

It is well known that the $J$-Integral is path independent because the same integral evaluated around a closed contour vanishes. The vanishing of the $J$-Integral around a close path under small strain assumptions is a consequence of the following two differential equations of equilibrium satisfied by the stress components [5]

$$
\begin{aligned}
& \frac{\partial \sigma_{x x}}{\partial x}+\frac{\partial \tau_{z x}}{\partial z}=0, \\
& \frac{\partial \tau_{z x}}{\partial x}+\frac{\partial \sigma_{z z}}{\partial z}=0 .
\end{aligned}
$$

The stress field in a laminated beam given by the shear deformable beam theory may not be accurate near the crack-tip, however they satisfy the above differential equations of equilibrium exactly. This is because the transverse shear stresses $\tau_{x z}$ in the beam are computed actually by substituting for $\sigma_{x x}$ and then integrating the first equilibrium equation. Thus the first of Eqs. (2) is satisfied. According to beam theories the shear stresses at a cross-section are proportional to the respective shear force $V$, which is constant along each ligament of the delaminated beam as well as in the intact beam ahead of the crack-tip. Thus the shear stresses $\tau_{x z}$ are independent of $x$ in each of the sub-laminates, and hence the first term in the second equilibrium equation is equal to zero. Since beam theories assume that $\sigma_{z z}$ are negligible, the second term is also equal to zero, and thus the second equilibrium equation is also satisfied. Then the $J$ Integral evaluated around the closed path $A B G H A$ (Fig. 1) using beam theory stresses is identically equal to zero. Since the integral along the horizontal paths are zero, we can show that $J_{A B}=J_{H G}$. Similarly we can show $J_{C D}=$ $J_{K L}$ and $J_{E F}=J_{M N}$. Thus we have moved the three vertical paths $A B, C D$ and $E F$ close to the crack-tip ( $H G, K L$ and $M N$ ) without affecting the value of $G$. The $J$-Integral evaluated around the paths 2, 3 and $1(H G K L M N)$ close to the crack-tip has been called the zero-volume $J$-Integral or zero-area $J$-Integral $[6,7]$ and $G$ is given by

$G=J^{(1)}+J^{(2)}+J^{(3)}$,

where the superscripts (1), (2) and (3), respectively, denote the paths $M N, H G$ and $K L$.

\subsection{G from strain energy densities}

Consider the $J$-Integral along path 1 in Fig. 1. Along this path $n_{x}=-1$ and $n_{z}=0$. Hence the Integral can be written as

$J^{(1)}=\int_{M}^{N}\left(-U_{0}+\sigma_{x x} u,_{x}+\tau_{z x} w,_{x}\right) \mathrm{d} s$.

We will add and subtract $\tau_{, z x} u_{, z}$ to the integrand in Eq. (4)

$$
\begin{aligned}
J^{(1)}= & \int_{M}^{N}\left(-U_{0}+\sigma_{x x} u,_{x}+\tau_{z x}\left(w_{, x}+u, z\right)\right) \mathrm{d} s \\
& -\int_{M}^{N} \tau_{z x} u,_{z} \mathrm{~d} s .
\end{aligned}
$$

The term $u_{z}$ can be identified as the rotation of the beam cross-section at the crack-tip, say, $\psi$, which is common to all the three paths 1,2 and 3. Further the sum of second and third terms in the first integral in Eq. (5) is equal to $2 U_{0}$. Hence Eq. (5) can be written as:

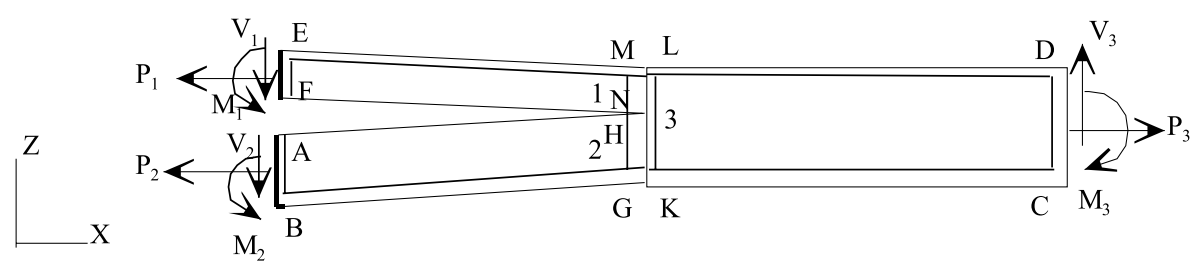

Fig. 1. Force and moment resultants in a delaminated beam. 
$J^{(1)}=\int_{M}^{N} U_{0} \mathrm{~d} s-\psi_{\mathrm{t}} \int_{M}^{N} \tau_{z x} \mathrm{~d} s$.

Eq. (6) can be further simplified as

$J^{(1)}=U_{\mathrm{L}}^{(1)}-\psi_{\mathrm{t}} V_{1}$

where $U_{\mathrm{L}}^{(1)}$ and $V_{1}$ are, respectively, the strain energy per unit length and shear force resultant at the cross-section 1 just behind the crack-tip. Similar results can be derived for paths 2 and 3 as follows

$J^{(2)}=U_{\mathrm{L}}^{(2)}-\psi_{\mathrm{t}} V_{2}$,

$J^{(3)}=-U_{\mathrm{L}}^{(3)}+\psi_{\mathrm{t}} V_{3}$.

It should be noted that $J^{(3)}$ in Eq. (8) switches signs because of change in the sign of $n_{x}$ from -1 to +1 for the path 3 . Adding all the three integrals and noting that the shear force resultants must satisfy the equilibrium condition $V_{1}+V_{2}=V_{3}$, we find that

$J=J^{(1)}+J^{(2)}+J^{(3)}=U_{\mathrm{L}}^{(1)}+U_{\mathrm{L}}^{(2)}-U_{\mathrm{L}}^{(3)}$.

Thus the energy release rate $G$ is the difference between the strain energy densities just behind and just ahead of the crack-tip. The strain energy density in the context of beams refers to strain energy per unit length of the beam, $U_{\mathrm{L}}$.

\section{3. $G$ in terms of crack-tip forces}

Consider a very small segment of the beam of length $2 \Delta x$ surrounding the crack-tip (Fig. 2). It will be convenient to shift the $x z$-coordinates such that the $x y$ plane coincides with the plane of delamination. Further, we will divide the laminate into four sub-laminates, two behind and two ahead of the crack-tip as shown in Fig. 2. Let the force and moment resultants near the cracktip in any sub-laminate be represented by a column matrix $\underline{F}$ such that $\underline{F}^{\mathrm{T}}=\lfloor P M V\rfloor$, where $P, M$ and $V$ are the axial force, bending moment and shear force resultants, respectively. An underscore denotes a matrix and a superscript $\mathrm{T}$ denotes transpose of the matrix. It should be noted that the force and moment resultants are resolved about the $x$-axis which coincides with the

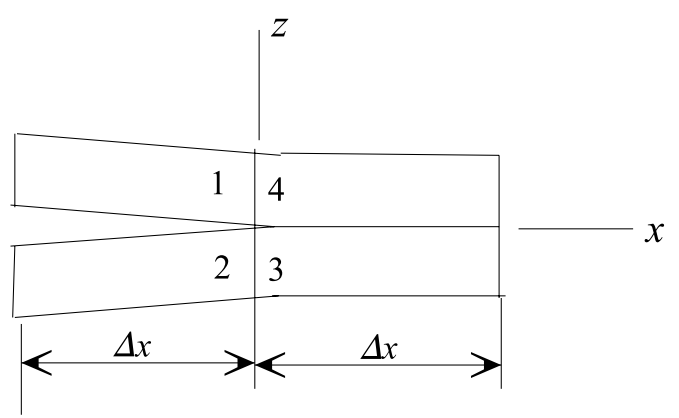

Fig. 2. Sub-laminates in a delaminated beam and the coordinate system. delamination plane. Thus there is an offset between the laminate mid-planes and the $x y$-plane. The force resultants in each sub-laminate are denoted by $\underline{F}_{1}, \underline{F}_{2}, \underline{F}_{3}$ and $\underline{F}_{4}$. The compliance matrix of the top and bottom sublaminates will be denoted by $\underline{C}_{\mathrm{t}}$ and $\underline{C}_{\mathrm{b}}$. The deformation in a sub-laminate is then given by

$\underline{e}=\underline{C F}$.

The deformations $\underline{e}$ are defined by

$\underline{e}^{\mathrm{T}}=\left\lfloor\begin{array}{lll}\varepsilon_{x 0} & \kappa_{x} & \gamma_{x z}\end{array}\right\rfloor$,

where the components of the deformations are the strain $\epsilon_{x 0}$ along the $x$-axis (not the sub-laminate mid-plane), rate of change of rotation $\kappa_{x}$ and the transverse shear strain $\gamma_{x z}$, respectively. The force resultants are related by the equilibrium conditions

$\underline{F}_{1}+\underline{F}_{2}=\underline{F}_{3}+\underline{F}_{4}$.

Further, since the sub-laminates 3 and 4 are intact (not delaminated) the deformations in them should be identical, i.e., $\underline{e}_{3}=\underline{e}_{4}$, and hence

$\underline{C}_{\mathrm{b}} \underline{F}_{3}=\underline{C}_{\mathrm{t}} \underline{F}_{4}$.

If $\underline{F}_{1}$ and $\underline{F}_{2}$ are given, then $\underline{F}_{3}$ and $\underline{F}_{4}$ can be calculated using the set of equations (12) and (13). The strain energy per unit length in any sub-laminate is given by

$U_{\mathrm{L}}=\frac{1}{2} \underline{F}^{\mathrm{T}} \underline{C} \underline{F}$.

Substituting Eq. (14) into Eq. (9) we obtain

$$
\begin{aligned}
G= & \frac{1}{2} \underline{F}_{1}^{\mathrm{T}} \underline{C}_{\mathrm{t}} \underline{F}_{1}+\frac{1}{2} \underline{F}_{2}^{\mathrm{T}} \underline{C}_{\mathrm{b}} \underline{F}_{2}-\frac{1}{2} \underline{F}_{3}^{\mathrm{T}} \underline{C}_{\mathrm{b}} \underline{F}_{3} \\
& -\frac{1}{2} \underline{F}_{4}^{\mathrm{T}} \underline{C}_{\mathrm{t}} \underline{F}_{4} .
\end{aligned}
$$

Using the relations (12) and (13) in Eq. (15) an interesting expression for $G$ can be derived

$G=\frac{1}{2}\left(\underline{F}_{4}^{\mathrm{T}}-\underline{F}_{1}^{\mathrm{T}}\right)\left(\underline{C}_{\mathrm{t}}+\underline{C}_{\mathrm{b}}\right)\left(\underline{F}_{4}-\underline{F}_{1}\right)$.

The term $\left(\underline{F}_{4}-\underline{F}_{1}\right)$ is actually the column matrix of forces transmitted through the crack-tip between the top and bottom sub-laminates, and can be called the cracktip forces, $\underline{F}_{\mathrm{c}}$. If a rigid link is used to connect the top and bottom crack-tip nodes in a finite element model

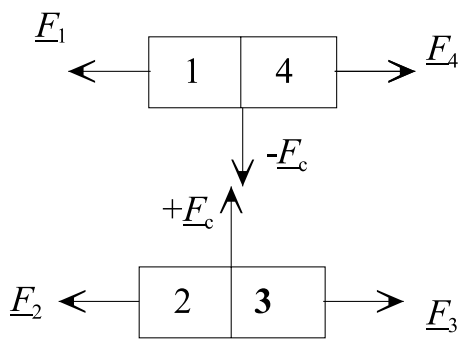

Fig. 3. Crack-tip forces acting between the top and bottom sub-laminates. 
(see Fig. 3), then the forces transmitted by the rigid link will be exactly equal to the above crack-tip forces. It may be noted that the crack-tip forces $\underline{F}_{\mathrm{c}}$ have three components, an axial force, a couple and a transverse force, corresponding to each degree of freedom of the crack-tip nodes, $u, \psi$ and $w$.

Another important implication of Eq. (16) is that although there are six independent forces $P_{1}$, $V_{1}, M_{1}, P_{2}, V_{2}$, and $M_{2}$ that can be applied to the two delaminated beam ligaments (see Fig. 1), $G$ depends only on the three crack-tip force components. If the forces $\underline{F}_{1}$ and $\underline{F}_{2}$ are such that $\underline{e}_{1}=\underline{e}_{2}$, i.e., $\underline{C}_{t} \underline{F}_{1}=\underline{C}_{b} \underline{F}_{2}$, then using Eqs. (12) and (13) one can show that $\underline{F}_{1}=\underline{F}_{4}$, and then $G=0$. If the forces on the top and bottom sublaminates 1 and 2 are such that they produce conforming deformations $\left(\underline{e}_{1}=\underline{e}_{2}\right)$, then the same forces act in sub-laminates 4 and 3 , respectively, producing conforming deformations $\left(\underline{e}_{3}=\underline{e}_{4}\right)$. Thus there is no need for any interaction between the top and bottom laminates at the crack-tip, and hence $G=0$.

\subsection{Virtual crack closure technique}

The VCCT technique has been used for plate and beam fracture problems by many researchers $[2,4,7,8]$. Originally the VCCT technique for plate-like structures was extended from the corresponding method used in continuum models [9]. In the present study we will derive the VCCT from the CTFM. The expression for $G$ in Eq. (16) can be written as

$G=\frac{1}{2} F_{\mathrm{c}}^{\mathrm{T}}\left(\underline{C}_{\mathrm{t}}\left(\underline{F}_{4}-\underline{F}_{1}\right)+\underline{C}_{\mathrm{b}}\left(\underline{F}_{2}-\underline{F}_{3}\right)\right)$,

where $\underline{F}_{\mathrm{c}}$ is the matrix of crack-tip forces, and Eq. (12) is used in deriving Eq. (17). Using the compatibility equation (13) in Eq. (17) we obtain

$G=\frac{1}{2} F_{\mathrm{c}}^{\mathrm{T}}\left(-\underline{C}_{\mathrm{t}} \underline{F}_{1}+\underline{C}_{\mathrm{b}} \underline{F}_{2}\right)$.

Since the matrix product $\underline{C F}$ denote deformations $\underline{e}$ we can write Eq. (18) as

$G=\frac{1}{2} F_{\mathrm{c}}\left\{\begin{array}{l}-u_{0, x}^{(1)}+u_{0, x}^{(2)} \\ -\psi_{0, x}^{(1)}+\psi_{0, x}^{(2)} \\ -w_{, x}^{(1)}+w_{, x}^{(2)}\end{array}\right\}$.

In deriving the last term of the column matrix in Eq. (19) we have used the fact that the beam rotation at the crack-tip is same for both ligaments 1 and 2, i.e., $\psi_{1}=\psi_{2}$. Multiplying and dividing the right-hand side of Eq. (19) by $-\Delta x$, where $\Delta x$ is a small length used in the virtual crack closure method, we obtain

$G=\frac{1}{2 \Delta x} F_{\mathrm{c}}^{\mathrm{T}}\left\{\begin{array}{c}\left(u_{0}^{(1)}-u_{0}^{(\mathrm{t})}\right)-\left(u_{0}^{(2)}-u_{0}^{(\mathrm{t})}\right) \\ \left(\psi^{(1)}-\psi^{(\mathrm{t})}\right)-\left(\psi^{(2)}-\psi^{(\mathrm{t})}\right) \\ \left(w^{(1)}-w^{(\mathrm{t})}\right)-\left(w^{(2)}-w^{(\mathrm{t})}\right)\end{array}\right\}$.
The superscript (t) in Eq. (20) denotes displacements and rotation at the crack-tip, and superscripts (1) and (2) denote, respectively, the displacements of the top and bottom ligaments at a distance $-\Delta x$ from the crack-tip. In deriving Eq. (20) we have used the finite difference approximation of the type

$u_{0, x}^{(1)}=\frac{u_{0}^{(\mathrm{t})}-u_{0}^{(1)}}{\Delta x}$.

Canceling the crack-tip displacements in Eq. (20) we obtain the equations for the virtual crack closure method as

$G=\frac{1}{2 \Delta x} F_{\mathrm{c}}^{\mathrm{T}}\left\{\begin{array}{c}\left(u_{0}^{(1)}-u_{0}^{(2)}\right) \\ \left(\psi^{(1)}-\psi^{(2)}\right) \\ \left(w^{(1)}-w^{(2)}\right)\end{array}\right\}$.

\section{Extension to delaminated plates}

In the case of delaminations in a plate the energy release rate $G$ varies along the delamination front. Consider a delaminated plate wherein the delamination front $\Omega$ is considered smooth. The sub-laminates behind the delamination front are designated as (1) and (2) and those ahead of the delamination front are designated as (3) and (4) (see Fig. 3). The delamination plane is chosen as the reference $x y$-plane. Assume that due to external loads the delamination front extends by an amount $\lambda(s)$, where $s$ is measured along the delamination front. Due to this advance of delamination front the sub-laminates (1) and (2) gain some areas whereas sub-laminates (3) and (4) lose equal amount of areas. Assuming the strain energy density (strain energy per unit area of the laminate) does not change very much due to the small amount of crack propagation, the change in strain energy of the plate can be computed as

$\Delta U=\oint_{s}\left(U_{A}^{(1)}+U_{A}^{(2)}-U_{A}^{(3)}-U_{A}^{(4)}\right) \mathrm{d} \lambda \mathrm{d} s$.

Let the point-wise energy release rate be represented by $G(s)$. Then by definition of the strain energy release rate under constant loads we obtain another expression for the change in the total strain energy of the plate

$\Delta U=\oint_{\Omega} G(s) \mathrm{d} \lambda \mathrm{d} s$.

If we have to obtain identical $\Delta U$ from Eqs. (23) and (24) for any arbitrary delamination propagation $\mathrm{d} \lambda(s)$, then the integrands in those equations should be identically equal to each other. Thus we obtain an expression for the point-wise $G(s)$ as

$G(s)=U_{A}^{(1)}+U_{A}^{(2)}-U_{A}^{(3)}-U_{A}^{(4)}$. 
One may note the similarity between Eqs. (25) and (9) derived for beams. Although Eq. (25) is an convenient expression for computing $G(s)$, accurate computation of the strain energy densities just ahead and behind the crack front seems to be difficult. Finite element programs compute the force and moment resultants at the Gauss quadrature points which are in the interior of plate elements adjoining the crack front. Extrapolation of the force resultants to the points along the crack front introduces some errors which are magnified in the computation of strain energy density $U_{A}$. Since Eq. (25) involves differences in the strain energy densities behind and ahead of the delamination front, the computed $G(s)$ has much larger errors. In order to avoid these errors an alternative method called CTFM is derived from Eq. (25).

We can use the same notation as we used for beams with the understanding that there are eight force and moment resultants, and eight deformation components (Whitney, 1987):

$$
\begin{aligned}
& \left\lfloor\underline{F}^{\mathrm{T}}\right\rfloor=\left\lfloor\begin{array}{llllllll}
N_{x} & N_{y} & N_{x y} & M_{x} & M_{y} & M_{x y} & Q_{x} & Q_{y}
\end{array}\right\rfloor, \\
& \left\lfloor\underline{e}^{\mathrm{T}}\right\rfloor=\left\lfloor\begin{array}{llllllll}
\varepsilon_{x 0} & \varepsilon_{y 0} & \gamma_{x y} & \kappa_{x} & \kappa_{y} & \kappa_{x y} & \gamma_{z x} & \gamma_{z y}
\end{array},\right.
\end{aligned}
$$

where $N, M$ and $Q$, respectively, are the inplane force, moment and shear force resultants. The laminate compliance matrix $[C]$ will be an $8 \times 8$ symmetric matrix, and it relates the force resultants and deformations:

$\underline{e}=\underline{C} \underline{F}=\left[\begin{array}{ccc}A & B & 0 \\ B & D & 0 \\ 0 & 0 & K\end{array}\right]^{-1}\{\underline{F}\}$,

where the $[A],[B]$ and $[D]$ are the classical $3 \times 3$ laminate stiffness matrices and $[K]$ is the $2 \times 2$ transverse shear stiffness matrix. In the context of plates the strain energy density is defined as strain energy per unit area of the plate and is given by

$U_{A}=\frac{1}{2} F^{\mathrm{T}} \underline{C F}$.

Using the procedures used in deriving Eq. (16) from Eq. (9) for delaminated beams, one can derive another expression for $G(s)$ from Eq. (25) as

$G=\frac{1}{2}\left(\underline{F}_{4}^{\mathrm{T}}-\underline{F}_{1}^{\mathrm{T}}\right)\left(\underline{C}_{\mathrm{t}}+\underline{C}_{\mathrm{b}}\right)\left(\underline{F}_{4}-\underline{F}_{1}\right)$.

Table 1

Elastic constants of materials used in the numerical examples

\begin{tabular}{lccc}
\hline $\begin{array}{l}\text { Elastic constants } \\
(\mathrm{GPa})\end{array}$ & Resin & Aluminum & $\begin{array}{l}\text { Graphite/ } \\
\text { epoxy }\end{array}$ \\
\hline$E_{1}$ & 3.4 & 71.0 & 134 \\
$E_{2}$ & 3.4 & 71.0 & 13.0 \\
$G_{12}$ & 1.3 & 27.3 & 6.40 \\
$v_{12}$ & 0.3 & 0.30 & 0.34 \\
\hline
\end{tabular}

As before, the term $\left(\underline{F}_{4}-\underline{F}_{1}\right)$ is the matrix of crack-tip forces. They also represent the jump in force and moment resultants that occur across the delamination front.

Sankar and Sonik [9] showed that three of the eight force resultants in a delaminated plate will be continuous across the delamination front. Assume a coordinate system such that the $x$-axis is normal to the crack front, $y$-axis is tangential to the crack front and $z$ is the thickness direction. Then the continuous force resultants are: $N_{y}, M_{y}$ and $Q_{y}$. Thus the jumps in these force resultants are zero, i.e.,

$N_{y}^{(4)}-N_{y}^{(1)}=N_{y}^{(2)}-N_{y}^{(3)}=0$,

$M_{y}^{(4)}-M_{y}^{(1)}=M_{y}^{(2)}-M_{y}^{(3)}=0$,

$Q_{y}^{(4)}-Q_{y}^{(1)}=Q_{y}^{(2)}-Q_{y}^{(3)}=0$.

Thus there will be only five components to the crack-tip forces: three forces in the $x, y$ and $z$ directions; two couples about the $x$ and $y$ axes, respectively. The three forces will be the jumps in $N_{x}, N_{x y}$ and $Q_{x}$ across the delamination front, either in the top laminates (1 and 4) or bottom laminates ( 2 and 3 ). The two crack-tip couples are the jumps in $M_{x}$ and $M_{x y}$. Since the jumps in $N_{y}, M_{y}$ and $Q_{y}$ are equal to zero and they do not contribute to the crack-tip forces, we can delete the second, fifth and seventh rows and columns in $C_{\mathrm{t}}$ and $C_{\mathrm{b}}$; we will denote them by $C_{\mathrm{t}}^{\prime}$ and $C_{\mathrm{b}}^{\prime}$. Then from Eq. (29) an expression of point-wise energy release rate can be derived as

$G(s)=\frac{1}{2} \underline{F}_{\mathrm{c}}^{\mathrm{T}}\left(\underline{C}_{\mathrm{t}}^{\prime}+\underline{C}_{\mathrm{b}}^{\prime}\right) \underline{F}_{\mathrm{c}}$,

where the crack-tip forces are given by

$$
\begin{aligned}
\left\lfloor\underline{F}_{\mathrm{c}}^{\mathrm{T}}\right\rfloor= & {\left[\left(N_{x}^{(4)}-N_{x}^{(1)}\right)\left(N_{x y}^{(4)}-N_{x y}^{(1)}\right)\left(M_{x}^{(4)}-M_{x}^{(1)}\right)\right.} \\
& \left.\times\left(M_{x y}^{(4)}-M_{x y}^{(1)}\right)\left(Q_{x}^{(4)}-Q_{x}^{(1)}\right)\right] .
\end{aligned}
$$

The compliance matrices $\underline{C}^{\prime}$ will take the form

$$
\underline{C^{\prime}}=\left[\begin{array}{lllll}
C_{11} & C_{13} & C_{14} & C_{16} & C_{18} \\
C_{13} & C_{33} & C_{34} & C_{36} & C_{38} \\
C_{14} & C_{34} & C_{44} & C_{46} & C_{48} \\
C_{16} & C_{36} & C_{46} & C_{66} & C_{68} \\
C_{18} & C_{38} & C_{48} & C_{68} & C_{88}
\end{array}\right],
$$

where the $C_{i j}$ are the coefficients of the full compliance matrix $\underline{C}_{\mathrm{t}}$ or $\underline{C}_{\mathrm{b}}$.

It should be mentioned that the crack-tip forces in Eq. (31) are line forces which vary along the delamination front. However in FE models discrete rigid links or multi-point constraints will be used along the delamination front. The forces in these rigid links should be divided by their spacing to obtain the crack-tip line forces. 


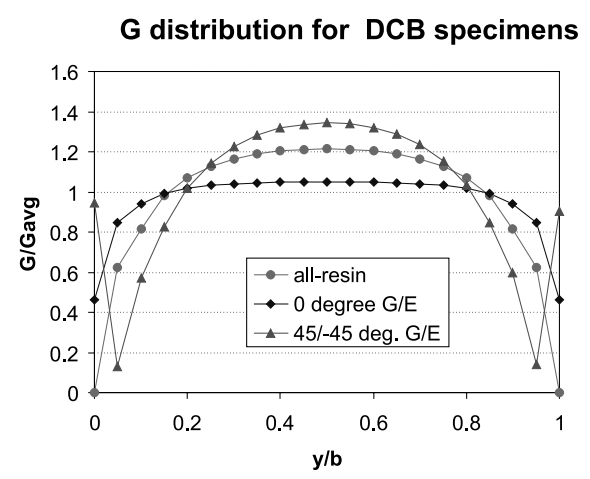

Fig. 4. Point-wise $G$ distribution in various specimens.

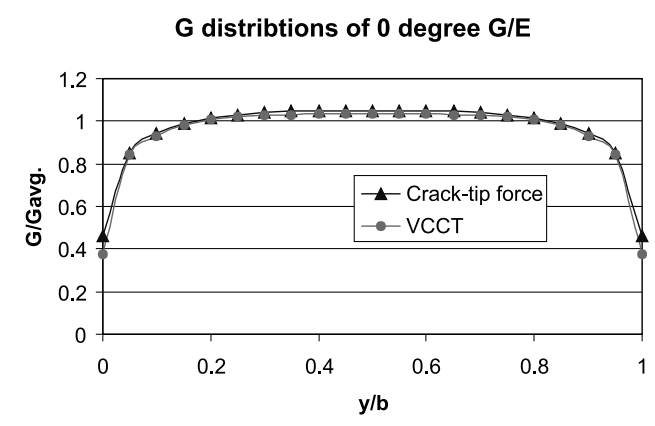

Fig. 5. Comparison of VCCT and CFTM.

\section{Results and discussion}

In order to verify the effectiveness of the proposed the crack-tip force method (CTFM) a double cantilever beam example was considered (Fig. 5). The dimensions of the DCB specimen are that used by Raju et al. [8] and are as follows: total length $101.6 \mathrm{~mm}$; delamination length $50.8 \mathrm{~mm}$; width $25.4 \mathrm{~mm}$; total specimen thickness $3.3 \mathrm{~mm}$; sub-laminate thickness $1.65 \mathrm{~mm}$. The material properties used are listed in Table 1 . In addition to unidirectional graphite/epoxy, a 16-layer angle ply laminate with the lay-up $[+45,-45]_{8}$ was also analyzed. This particular lay-up is of academic interest only for its curing at temperatures higher than the room temperature will cause warpage. In the numerical examples the transverse force applied to each ligament of the DCB is $1 \mathrm{~N} / \mathrm{m}$.

The normalized energy release rate values for the various specimens computed using the CFTM are shown in Fig. 4. These results compare well with the 3-D analysis results of Raju et al. [8]. A comparison of $G$ computed by the virtual crack closure technique (VCCT) and CTFM is shown in Fig. 5. The comparison is very good for the example considered.

A new method called CTFM is derived for computing point-wise energy release rate along the delamination front in delaminated plates. Actually the method can be derived from the VCCT or the previously derived strain energy density method. However the CTFM is computationally simple as $G$ is computed using the forces transmitted at the crack-tip between the top and bottom sub-laminates and the sub-laminate properties. Although the strain energy density method is elegantly simple and theoretically exact, accurate computation of strain energy densities on either side of the crack front may not be possible. Future work will involve application of the aforementioned methods to general laminates containing internal delaminations, free edge delaminations and debonded stiffened panels.

\section{Acknowledgements}

This study was supported by NASA Langley Research Center Grants (NAG1-1887, 2000 and 2177) to the University of Florida. The authors are thankful to Professor R.T. Haftka for his many suggestions.

\section{References}

[1] Sankar BV, Hu S. Dynamic delamination propagation in composite beams. J Compos Mater 1991;25:1414-26.

[2] Zheng S, Sun CT. A double-plate finite-element model for the impact-induced delamination problem. Compos Sci Technol 1995; 53:111-8.

[3] Olsson R. A simplified improved beam analysis of the DCB specimen. Compos Sci Technol 1992;43:329-38.

[4] Sankar BV, Rao VS. A plate finite element for modeling delaminations. J Reinf Plast Compos 1993;12:227-36.

[5] Anderson TL. Fracture mechanics - fundamentals and applications. Boca Raton, FL: CRC press; 1995.

[6] Farris TN, Doyle JF. Estimating dynamic stress intensity factors in a length-wise cracked beam using remote data. In: Engineering sciences preprint ESP2588026. Blacksburg, VA: Society of Engineering Sciences; 1988.

[7] Sankar BV. A finite element for modeling delaminations in composite beams. Comput Struct 1991;38:239-46.

[8] Raju IS, Shivakumar KN, Crews JH. Three-dimensional elastic analysis of a composite double cantilever beam specimen. AIAA J 1998;26:1493-8.

[9] Sankar BV, Sonik V. Point-wise energy release rate in delaminated plates. AIAA J 1995;33:1312-8. 